\title{
Pharmazeutika im Trinkwasser
}

\author{
Eine soziologische Annäherung an das Problem \\ der Nebenfolgen in der Krankenbehandlung
}

Christian Büscher, Institut für Technikfolgenabschätzung und Systemanalyse (ITAS), Karlsruher Institut für Technologie (KIT), Karlstr.11, 76133 Karlsruhe (buescher@kit.edu)

Das soziale System der Krankenbehandlung erbringt nicht nur wesentliche Leistungen in der Gesellschaft, sondern externalisiert systemisch unerwünschte Folgen, z. B. Pharmazeutikarückstände in Gewässern. Diese nicht-intendierten Nebenfolgen resultieren aus unzähligen, alltäglichen Einzelhandlungen, die durch soziale Mechanismen des Umgangs mit Unsicherheit erst ermöglicht werden. Zur Entwicklung von Strategien zur Reduzierung von Pharmazeutikarückständen im Trinkwasser muss die Wirkmächtigkeit von Mechanismen wie Geldzahlungen, Autorität oder Vertrauen besser verstanden werden. Diese bewirken das Zustandekommen von Handlungen, lassen sich aber schwerlich zur Steuerung von erwünschtem Verhalten instrumentalisieren.

\section{Pharmaceuticals in drinking water}

A sociological approach to the problem of unintended consequences of medical treatment

The social system of medical treatment does not only provide essential societal services, it also externalizes systemically undesirable effects, e. g., pharmaceuticals in the aquatic environment. These unintended consequences result from countless individual actions that arise from social mechanisms of dealing with uncertainty. In order to develop strategies to reduce pharmaceuticals in drinking water, it is important to better understand the efficacy of mechanisms such as monetary payments, authority, and trust. Those allow for the emergence of actions, but can hardly be used to control desired actions.

Keywords: medical treatment, doctor-patient interaction, uncertainty, risk, ecological consequences

This is an article distributed under the terms of the Creative Commons Attribution License CCBY 4.0 (https://creativecommons.org/licenses/by/4.0/)

https://doi.org/10.14512/tatup.27.3.51

Submitted: 01. 02.2018. Peer reviewed. Accepted: 14.09.2018

\section{Einleitung}

In dieser soziologischen Perspektive auf das Problem „Medikamentenrückstände im Trinkwasser" sollen Argumente für ein neues, zukünftig empirisch zu fundierendes, Forschungsdesign vorgestellt werden, um die Möglichkeiten und Chancen einer generellen Verringerung des Konsums von Pharmazeutika abzuschätzen. Pharmazeutika im Trinkwasser werden hier als eine Nebenfolge der organisierten, technisierten und verwissenschaftlichten Krankenbehandlung verstanden. Diese verursacht, trotz enormer Leistungsfähigkeit, auch weitere Nebenfolgen, wie zu hohe Kosten, überlastetes Personal, resistente Keime oder Behandlungsfehler. Von den üblichen Vorschlägen, eine Verringerung des Pharmazeutikakonsums sei durch Aufklärung, Informierung oder Bewusstsein von Personen(gruppen) zu erreichen, soll zunächst Abstand genommen werden. Vielmehr wird diskutiert, ob neue Einsichten zu erwarten sind, wenn soziale Mechanismen der Ermöglichung von Krankenbehandlung in den Mittelpunkt der Analysen gestellt werden. Ansatzpunkt

\section{Die nachfolgenden Überlegungen schließen an interdisziplinäre} Forschungsarbeiten an, die Pharmazeutika im Trinkwasser als systemi-
sches Risiko charakterisiert haben.

ist die kontraintuitive These aus der Grundlagenforschung in der Medizinsoziologie, dass Krankenbehandlung eine (notwendige) Zumutung sei. Krankenbehandlung ist also zunächst als unwahrscheinlich einzuschätzen (Fuchs 2006, S. 28; Begenau 2009, S. 25), weil alle Beteiligten neben der Aussicht auf erfolgreiche Heilung bzw. Linderung immer auch die Erwartung mög- 
licher negativer Konsequenzen verarbeiten müssen. Krankenbehandlung ist immer mit Unsicherheit und Risiko behaftet. Somit bedarf auch die Medikamentenverschreibung als grundlegender Bestandteil der Krankenbehandlung bestimmter Mechanismen, die Unsicherheiten insoweit reduzieren, dass Entscheiden möglich ist (Fuchs 2006, S. 28; Koch und Sawicki 2009). Mit diesem abstrakten, aber grundlegenden Gedanken sollen gängige Rationalitäts- und Vernunftsprämissen hinterfragt werden, die auf Verhaltensänderungen für eine Reduzierung von Medikamentenrückständen im Trinkwasser durch Informierung hoffen.

Pharmazeutika gelangen über den Weg der unsachgemäßen Entsorgung oder als Rückstände in den Ausscheidungen von $\mathrm{Pa}$ - lage wird eine ganze Bandbreite von Maßnahmen diskutiert, die sowohl technische Lösungsansätze, z. B. Medikamentendesign (Kümmerer und Clark 2016) oder Filtertechnik in Kläranlagen als auch Verhaltensansätze beinhalten (für eine Übersicht: Hillenbrand et al. 2016, S. 189 ff.). Letztere zielen, neben der richtigen Entsorgung von nicht verbrauchten Medikamenten, auf die Verringerung des Medikamentenkonsums ab (Keil et al. 2008, S. 358), z. B. durch eine nachhaltige Verschreibungspraxis (Pahl 2015; civity 2017, S.39).

Im Folgenden wird kurz auf grundlegende Aspekte der Leistungserwartungen und Motivation eingegangen, um anschlieBend anhand der Mechanismen Geldzahlung, Vertrauen, Auto-
tientinnen/Patienten in den Wasserkreislauf (Schulte-Oehlmann et al. 2007). Sie sind zwar im Design auf ein Mindestmaß an chemischer Stabilität ausgelegt, um die erwünschte Wirkung im Organismus zu erreichen, eine Restwirkung verbleibt aber auch nachdem die Wirkstoffe im Organismus metabolisiert wurden. Da auch modernste Filteranlagen das Trinkwasser nicht vollständig von Medikamentenrückständen reinigen können, werden diese von der Bevölkerung in Mikrodosierungen wiederaufgenommen. ${ }^{1} \mathrm{Ob}$ und welche Auswirkungen die dauerhafte indirekte Exposition auf den menschlichen Organismus hat, ist noch nicht bekannt (Webb et al. 2003; Touraud et al. 2011) und wird aktuell (noch) als ungefährlich eingeschätzt (Klauer 2018, S. 23). Dennoch ruft allein der Umstand der unfreiwilligen Exponierung Besorgnis hervor und verlangt nach langfristigen Strategien (Kümmerer et al. 2015). Dementsprechend erfahren Pharmazeutika im Trinkwasser aktuell einige Aufmerksamkeit, z. B. durch das Interreg-Projekt noPills (Pahl 2015), eine Studie im Auftrag des Bundesverbandes der Energie- und Wasserwirtschaft (civity 2017), durch den TAB-Arbeitsbericht Nr. 178 (Klauer 2018) ${ }^{2}$ oder durch eine internationale Metastudie (Beek et al. 2016).

Die nachfolgenden Überlegungen schließen an interdisziplinäre Forschungsarbeiten an, die Pharmazeutika im Trinkwasser als systemisches Risiko charakterisiert haben, also als Ergebnis von ,decentralized, intentional processes and cycles in a system which lead to systemic, cumulative production of hazard" (Keil et al. 2008, S. 355). Als Reaktion auf diese Gefährdungs-

1 Umweltrelevante Wirkstoffe sind den Laien als Antibiotika (antibakterielle Mittel, Antiinfektiva, bakterientötende Mittel), Antiepilektika, Lipidsenker (Cholesterinsenker), Sexualsteroide oder Zytostatika (Zellwachstum hemmende Stoffe, Krebsmittel, Mittel gegen Autoimmunerkrankungen oder als Stoffe zur Hemmung der Zellteilung) bekannt (Schulte-Oehlmann et al. 2007, S. 168). 2 Zum Zeitpunkt der Einreichung dieses Artikels war der Bericht noch nicht veröffentlicht. rität aufzuzeigen, wie Unsicherheitsabsorption wirken kann und wie die Operationalisierung von Behandlungserwartungen und -erbringung in der Arzt-Patienten-Interaktion vor sich geht. Damit kann besser abgeschätzt werden, wie diese Mechanismen auf die Verringerung oder Erhöhung der Medikamenteneinnahme einwirken könnten.

\section{Leistungserwartungen an und Motivation zur Krankenbehandlung}

An den Komplex der Krankenbehandlung richten sich Leistungserwartungen der arbeitsteiligen Funktionserfüllung der ambulanten und stationären ärztlichen Versorgung, der Krankenund Pflegeversicherung, der Arzneimittelversorgung sowie der ambulanten und stationären Pflege (Wendt 2003; Simon 2005; Paul und Schmidt-Semisch 2010). Dazu sind in den letzten Jahrzehnten eine Vielzahl von Arbeiten entstanden, die sich um die Klärung des Systemstatus und funktionaler Probleme der Systemerhaltung verdient gemacht haben (Vogd 2005; Fuchs 2006; Pelikan 2009; Stollberg 2009). Für uns sind im Folgenden zwei Aspekte von besonderer Bedeutung.

\section{Demografische Trends und individuelle Ansprüche}

Die konkrete Ausgestaltung von zulässigen Ansprüchen und Behandlungsprogrammen beeinflusst den Konsum von Pharmazeutika in direkter Weise. Anspruchshaltung und Programmierung werden durch demografische Trends und soziale Erwartungsbildung beeinflusst.

Als Begründung für die umfassende Steigerung der Aktivitäten in Forschung und Entwicklung sowie in der Produktion und Konsumption von Medikamenten werden Veränderungen sowohl in der demografischen Struktur als auch in den Bedürf- 
nissen und Ansprüchen der Bevölkerung angeführt (Dingermann 2006; civity 2017). Zwei Faktoren werden dabei hervorgehoben.

Erstens korrelieren die Altersverteilung in der Gesellschaft und die Entwicklung im Arzneimittelgebrauch wechselseitig. Durch gestiegene Lebenserwartung und die gesunkene Geburtenrate wächst der Anteil der Personen, die 65 Jahre und älter sind, schneller als der jeder anderen Altersgruppe. Das hat Konsequenzen für die medizinische Versorgung, da bei älteren und sehr alten Menschen typischerweise mehrere Leiden gleichzeitig auftreten. In der Folge kommt es zur erschwerten Diagnosestellung, zur verminderten Therapietreue (compliance) so-
Unsicherheiten über die Folgen der Behandlungen sind auf beiden Seiten (der Experten und der Laien) gegeben und müssen abgebaut werden. Aufgrund der fortgesetzten Verwissenschaftlichung und Technisierung der Krankenbehandlung kommt es zu einem eklatanten Auseinanderdriften von klinischer Diagnose aufseiten der Profession und den lebensweltlichen Erfahrungen aufseiten der Patientinnen/Patienten (Stevenson 2006, S. 226). Auch medizinische Experten unterliegen der Bürde, mit Nichtwissen umgehen $\mathrm{zu}$ müssen, nur fällt es dem medizinischen Laien noch weitaus schwerer, die daraus resultierenden Unsicherheiten auszublenden (Vogd 2005, S. 8).

Nach Vogd (2005, S. 244 f.) und Stollberg (2009, S. 206)

\section{Für eine Reduzierung des Medikamentenverbrauchs muss auch die Arzt-Patienten-Interaktion analysiert werden, in der die Medikamentenvergabe konkret umgesetzt wird.}

wie insgesamt zu einem erhöhten Bedarf an medizinischen und pflegerischen Behandlungsressourcen (Rodrig und Wiesemann 2004; Dingermann 2006, S. 7; civity 2017, S. 6).

Zweitens kann der gestiegene Medikamentenkonsum durch die individuelle Anspruchssteigerung erklärt werden. Sowohl die Vorsorgemedikation als auch die Lifestyle-Medikation werden verstärkt in Anspruch genommen. Zugespitzt formuliert kommt es vermehrt zur ,pharmakotherapeutischen Behandlung Gesunder“ (Dingermann 2006, S. 11). Dem liegt ein zirkulärer, sich selbst verstärkender Mechanismus zugrunde: Zum einen erweisen sich individuelle Bedürfnisse, Präferenzen und Zahlungsbereitschaften als Determinanten für die Entwicklungen im System der Krankenbehandlung als demand pull, zum anderen werden diese wiederum erst von den wissenschaftlichen, medizintechnischen und pharmakologischen Möglichkeiten als supply push geweckt (Dingermann 2006). Bratan und Wydra (2013, S. 156) sehen in diesem „Wissenschafts- und Technikpush“ eine zu korrigierende Fehlorientierung.

\section{Motivation zur Krankenbehandlung}

In der modernen Gesellschaft nimmt Krankheit die Form des wissenschaftlich informierten medizinischen Befundes an, der es erlaubt, die Patientin/den Patienten als Fall zu behandeln, woraufhin Methoden und technische Mittel zur Wiederherstellung eines Normalzustandes zum Einsatz kommen. Gemäß unserer Eingangsdefinition von Krankenbehandlung als (notwendiger) Zumutung veranlasst das Erleben von Unwohlsein und Schmerz Patientinnen/Patienten dazu, eine Reihe unwahrscheinlicher Prozeduren zu erdulden: die Behandlung selbst, die Offenlegung des Körpers gegenüber nicht intimen, fremden Personen oder auch die Fortführung der Behandlung, selbst wenn das Erleben von Unwohlsein und Schmerz nachlässt oder verschwindet (Fuchs 2006, S. 28). kommt hier die Funktion der evidence-based medicine zum Tragen, indem der medizinische Experte bei Unsicherheiten der Behandlung auf spezielle fachorganisatorisch legitimierte Leitlinien zurückgreifen kann (Koch und Sawicki 2009, S. 1). Medizinischen Laien stehen solche Instrumentarien nicht zur Verfügung, sie müssen dem Befund vertrauen, wenn sie behandelt werden wollen, und dann mehr oder weniger die Programmausführung erdulden.

Wenn eine generelle Reduzierung des Medikamentenverbrauchs bewirkt werden soll, dann muss neben exogenen Faktoren (Demografie, Anspruchshaltung) und endogenen Programmierungen (evidenzbasierten Leitlinien) auch die Arzt-PatientenInteraktion analysiert werden, in der die Medikamentenvergabe konkret umgesetzt wird (siehe dazu auch Pahl 2015, S. 16).

\section{Die Arzt-Patienten-Interaktion}

In der Interaktion zwischen Ärztinnen/Ärzten und Patientinnen/Patienten findet die Operationalisierung von Leistungserwartung und -erbringung ihren Ausdruck, wenn individuelle Ansprüche auf Strukturen (die Organisation des Erstkontakts) und Programme (die rationalisierte evidenzbasierte Medizin) treffen und Festlegungen getroffenen werden, die alle weiteren Vorgänge konditionieren (Therapien, Überweisung, Abrechnung etc.). Die Ärztin/der Arzt erlebt nicht dasselbe wie die Patientin/der Patient und muss trotzdem handeln, und die Patientin/ der Patient hat nicht dasselbe Handlungsvermögen wie die Ärztin und muss dennoch die Behandlung erdulden, um eine Heilung zu erreichen (Toombs 1992, S. 118). Im Folgenden nennen wir drei Mechanismen, die im Arzt-Patienten-Verhältnis ermöglichende und einschränkende Wirkungen auf Behandlungsformen und auf den Medikamentenverbrauch haben. 


\section{Geldzahlungen zur Motivation eines wechselseitigen Austauschs}

Geldzahlungen motivieren die Behandlung aufseiten der Leistungserbringer und gleichzeitig die Anspruchshaltung aufseiten der Leistungsempfänger, für die geleisteten Zahlungen die bestmögliche Behandlung zu erhalten. Mitglieder von Solidargemeinschaften können leicht den Anspruch entwickeln, mindestens so viele Leistungen zu erhalten, wie es ihren Beiträgen in die Solidarkassen entsprechen würde. Niemand strebt ernsthaft einen Krankenzustand an, aber wenn dieser Fall eintritt, dann lässt sich durch den Mechanismus der Geldzahlung kaum eine freiwillige Selbstbeschränkung nur auf das Nötigste abringen (abgedämpft durch positive Anreize wie Prämien oder negative Anreize wie Zuzahlungen). Auch aufseiten der Leistungserbringer geraten Anreizstrukturen in die Diskussion, die eine Behandlungssteigerung erwarten lassen. Zum einen werden niedergelassene Ärzte und Kliniken gezwungen, durch die Abrechnung von Einzelleistungen das Kriterium der „Rentabilität" in das Behandlungskalkül einzubeziehen, was eher eine Entwicklung in Richtung optimale Auslastung von Personal und Apparatur erwarten lässt (Vogd 2005). Zum anderen offerieren am Markt konkurrierende Versicherungsgeber potenziellen Beitragszahlern immer mehr Leistungsversprechen (Offermanns 2009). In diesem Sinne muss damit gerechnet werden, dass über Art und Umfang der Krankenbehandlung nicht allein nach Kriterien des medizinisch Notwendigen, sondern ebenso nach dem medizinisch Möglichen entschieden wird. Solvente Patientinnen/Patienten fragen nach, was je nach Situation auf dem Medizinmarkt angeboten wird. Patientinnen/Patientenen sind nicht mehr nur Erduldende, sondern ebenso handelnde Kunden auf dem Medizinmarkt, welche ,die Ungleichheit des Wissens zwischen Arzt und Patientin/Patient durch Wahlhandlungen auf dem medizinischen Markt" kompensieren (Stollberg 2007, S. 359).

\section{(System)Vertrauen zur Überwindung zeitlich bedingter Unsicherheiten}

Vertrauen zielt auf die Überbrückung der zeitlichen Differenz von Gegenwart und Zukunft, die uns dazu zwingt, dass wir uns in der Gegenwart auf Situationen einlassen, ohne dass wir die in der Zukunft anfallenden Folgen schon kennen. Deshalb ist Vertrauen ein wesentlicher sozialer Mechanismus in der Interaktion von Ärztin/Arzt und Patientin/Patient. Vertrauen ist dabei keine mentale Kategorie. Vielmehr geht es um den risk taking act in der sozialen Beziehung (Skinner et al. 2013). Die Patientin/der Patient kann (und muss) gerade bei der Krankenbehandlung auch widerwillig vertrauen, und zwar der Profession allgemein oder einzelnen Personen. Die Ärztin/der Arzt wiederum muss einen Vertrauensvorschuss durch den Hinweis auf den Standesethos der Profession einfordern, um Konsultation zu ermöglichen. Nur so kann die Patientin/der Patient im Folgenden die Rolle der/des Schutzbefohlenen und Konsultierenden einnehmen. Dieses Ethos blendet andere Codierungen - vor allem monetäre Aspekte - aus: Thematisiert wird zunächst nur Heilung.
Kommt hingegen der Verdacht auf, auch andere Kriterien, zum Beispiel die Wirtschaftlichkeit eines Krankenhauses oder einer Praxis, könnten eine dominierende Rolle einnehmen, dann gerät dieser Vertrauensvorschuss in Gefahr (Wendt 2003, S. 273).

Vogd (2005) diagnostiziert hier Verschiebungen innerhalb der Rationalität moderner Behandlungseinrichtungen, in denen eine auf langfristiger Vertrautheit basierende Beziehung zwischen Ärztin/Arzt und Patientin/Patient nicht mehr vorgesehen ist und einem „Systemvertrauen“ weichen muss (Shapiro 1987; Luhmann 1988). Die Bedeutung des Professionellen schwindet zugunsten arbeitsteiliger Organisation (Programm aus ärztlicher Einstellung der Medikation, Labor-Monitoring, therapeutische Anwendungen durch Spezialisten, Stationsdisziplin). Als funktionale Äquivalenz zum vertrauten Arzt wird versucht, Vertrauen in unpersönliche Medikamentenmarken sowie in Diagnose- und Behandlungsprogramme bzw. -techniken aufzubauen, zum Beispiel über Zertifizierungen, Rekurs auf Tradition, Leitbilder, evidenzbasierte Therapie- und Pflegeangebote (Vogd 2005, S. 255). Wenn aber Vertrauen auf Image basiert, dann treffen sich Kunden und Dienstleister in komplementären Rollen. Erstere wählen nach qualitativen und quantitativen Leistungsaspekten aus, was Letztere in maximaler Hinsicht anbieten, um ein erfolgreiches Image überhaupt erst einmal herstellen und dann aufrechterhalten zu können. In dieser Hinsicht agieren Ärzte und Patientinnen/Patienten als „Bündnispartner“, behauptet Vogd: Die aufgeklärten Patientinnen/Patienten können dazu genutzt werden, der von außen bezweckten Limitierung des Systems entgegenzuwirken, wenn diese die Bezahlung von teuren und aufwendigen Therapien bei den Krankenkassen einfordern oder selbst übernehmen (ebd., S. 255). Auch hinsichtlich des Vertrauensmechanismus wirken demnach Kräfte tendenziell in Richtung Ausdifferenzierung und Ausweitung von Krankenbehandlung.

\section{Die Unterstellung von Autorität zur Überwindung von Wissensdifferenzen}

Überall dort, wo eine Wissensdifferenz die Situation prägt, kann die Unterstellung eines Erläuterungspotenzials seitens des Experten, das von dem Laien nicht in Anspruch genommen wird, Komplexität reduzieren und Zeit einsparen (Friedrich 1958, S. 47). Deshalb ist Autorität in der Arzt-Patienten-Interaktion ein weiterer wichtiger Mechanismus, der die Übernahme von Informationen ohne langwierige Erläuterung erlaubt. Autorität zielt also auf eine sachliche Differenz von Wissen und Nichtwissen, und diese Differenz verschiebt sich durch aktuelle Entwicklungen, weil die medizinische Profession die alleinige Kontrolle über das medizinische Wissen zu verlieren scheint. Patientinnen/Patienten erhalten durch die Veröffentlichung von medizinischem Wissen durch zahlreiche Verbreitungsmedien einen umfangreichen Zugang zu Informationen. Vor allem das Internet spielt eine große Rolle, da es alternative Expertisen bereitstellt oder die Möglichkeit des anonymen Austauschs mit anderen von Krankheit Betroffenen erlaubt (O'Grady et al. 2008). Dieser Informationszugang kann die Autoritätsunterstellung unterminie- 
ren und zur Infragestellung von Diagnosen und Therapievorschlägen motivieren. Stevenson (2006, S. 238 f.) spricht deshalb bereits von einem geänderten Rollenverständnis aufseiten der Leistungsempfänger als consumerist im Gegensatz zur/zum passiven Patientin/Patienten. Dies kann den Wechsel zu einem ,passenden“ Arzt bzw. eine verstärkte Selbstmedikation, aber auch eine Ablehnung von Medikamenteneinnahmen zur Folge haben. Das bedeutet, der Mechanismus der Autorität kann wiederum in beide Richtungen wirken: Ausweitung und Einschränkung.

Aus diesen knapp gehaltenen Ausführungen lässt sich schlie-
Es stehen aber kaum empirische Forschungen zur Verfügung, die soziale Mechanismen in ihr Forschungsdesign aufgenommen haben. Das noPills-Projekt ist (meines Wissens nach) die einzige Ausnahme. Im Abschlussbericht wird zum Beispiel von den ambivalenten Ergebnissen hinsichtlich monetärer Steuerungsversuche berichtet: „A high price could make a product either more or less attractive to buy“ (Pahl 2015, S. 48). Mit Bezug auf Vertrauen als Element der Steuerung des Medikamentenkonsums ist eine Vielzahl von Adressaten von Interesse, da Patientinnen/Patienten nicht nur auf Ärztinnen/Ärzte, sondern

\section{Zukünftige empirische TA-Studien zur Reduzierung des Medikamentenkonsums sollten auch soziale Mechanismen als Systemmechanismen beleuchten.}

ßen, dass die vorgestellten sozialen Mechanismen auf der Ebene der Interaktion Lösungen für Probleme der Unsicherheitsabsorption darstellen, die im Weiteren eine Systemleistung zu erbringen erlauben, aber hinsichtlich der Kontrolle von individuellen Behandlungsansprüchen schwer zu instrumentalisieren sind. ${ }^{3}$

\section{Fazit}

Das soziale System der Krankenbehandlung verleiht sich durch Solidar-, Bedarfsdeckungs- und Sachleistungsprinzipien Einschränkung der eigenen Aktivitäten (Simon 2005, S. 35 ff.). Wir wissen aber, dass Versicherungen und Versicherte das Leistungsspektrum durch Angebot und Nachfrage sukzessive erweitern. Auch wird Bedarf generiert, indem durch verschärfte Kriterien ehemals Gesunde zu Kranken umdefiniert werden (Schlimpert 2018). Zusätzliche Leistungen werden durch den wissenschaftlich-technischen Fortschritt permanent hinzuerfunden (Bratan und Wydra 2013). Das System selbst tendiert eher zur Überhöhung seiner selbst, also zur Hypostasierung der eigenen Funktion. Die Stilisierung der modernen Gesellschaft als „Gesundheitsgesellschaft“ kann als Indiz dafür gewertet werden (Pelikan 2009).

In aktuellen Studien zur Reduzierung des Medikamentenkonsums werden eine Vielzahl von Regulierungs- und Steuerungsversuchen - monetärer oder rechtlicher Art - diskutiert.

3 Ein weiterer Diskussionsstrang zielt auf langfristige, präventive, die Lebensführung betreffende Strategien, die eine Nutzung von Medikamenten eindämmen könnten. Dem können wir an dieser Stelle leider nicht nachgehen. Nur so viel: Präventionserwartungen erzwingen Entscheidungssituationen, hinsichtlich der Änderung von Lebensstilen oder der Inanspruchnahme von Vorsorgeuntersuchungen bzw. vorsorglichem Technikeinsatz (Leanza 2010, S. 259). Die Verhaltensänderung, die erst langfristig Erfolge zeitigen wird, steht der kurzfristig relativ sicher wirkenden Behandlung mit Medikamenten gegenüber. auch auf Freunde und Verwandte als Informationsressource zurückgreifen (ebd., S. 16). Versuche, Autorität als Steuerungsmechanismus zu nutzen, können an dem Widerstand der Patientinnen/Patienten scheitern: ,distrust and negative experience predominate in authoritative relationships.“ (ebd., S. 48)

Diesem Beispiel folgend, sollten zukünftige empirische TA-Studien sich darum bemühen, soziale Mechanismen - wie in dieser Ausarbeitung angedeutet - als Systemmechanismen zu beleuchten: Geldzahlung, Vertrauen, Autorität entfalten ihre Wirkung in diesem Fall gerade in Richtung Geschwindigkeitsvorteile, Minderung der Reibung bzw. Absorption von Unsicherheit. Wenn ökologische Rücksichtnahmen, z. B. hinsichtlich der Qualität des Trinkwassers, in die systemischen Vorgänge einbezogen werden sollen, um generelle Verhaltensänderungen zu bewirken (Verschreibungspraktiken, Einnahmegewohnheiten, etc.), dann müssen dazu solcherart Mechanismen untersucht und besser verstanden werden. Appelle an die guten Absichten Einzelner sind vermutlich funktional im Nachteil.

\section{Literatur}

Beek, Tim aus der et al. (2016): Pharmaceuticals in the environment. Global occurrences and perspectives. In: Environmental Toxicology and Chemistry 35 (4), S. 823-835.

Begenau, Jutta; Schubert, Cornelius; Vogd, Werner (2009): Einleitung. Die ArztPatient-Beziehung aus soziologischer Sicht. In: Jutta Begenau, Cornelius Schubert und Werner Vogd (Hg.): Die Arzt-Patient-Beziehung. Stuttgart: Kohlhammer, S.7-33.

Bratan, Tanja; Wydra, Sven (2013): Technischer Fortschritt im Gesundheitswesen. Quelle für Kostensteigerungen oder Chance für Kostensenkungen? Arbeitsbericht Nr.157. Berlin: Büro für Technikfolgen-Abschätzung beim Deutschen Bundestag (TAB).

civity (2017): Arzneimittelverbrauch im Spannungsfeld des demografischen Wandels. Die Bedeutung des wachsenden Medikamentenkonsums in Deutschland für die Rohwasserressourcen. Berlin: civity Management Consultants im Auftrag des Bundesverband der Energie- und Wasserwirtschaft e. V. 
Dingermann, Theo (2006): Demographische Entwicklung und Heilmittelverbrauch. In: Fritz Frimmel und Margit Müller (Hg.): Heil-Lasten. Berlin: Springer, S. 3-28.

Friedrich, Carl (1958): Authority, reason and discretion. In: Carl Friedrich (Hg.): Authority. Cambridge, Mass.: Harvard Univ. Press, S. 28-48.

Fuchs, Peter (2006): Das Gesundheitssystem ist niemals verschnupft. In: Jost Bauch (Hg.): Gesundheit als System. Systemtheoretische Beobachtungen des Gesundheitswesens. Konstanz: Hartung-Gorre Verlag, S. 21-38.

Hillenbrand, Thomas et al. (2016): Maßnahmen zur Verminderung des Eintrages von Mikroschadstoffen in die Gewässer. Phase 2. Dessau-Roßlau: Umweltbundesamt.

Keil, Florian; Bechmann, Gotthard; Kümmerer, Klaus; Schramm, Engelbert (2008): Systemic risk governance for pharmaceutical residues in drinking water. In: GAIA 17 (4), S. 355-361.

Klauer, Bernd (2018): Arzneimittelrückstände in Trinkwasser und Gewässern. Medizinische Wirkstoffe in Trinkwasser und Gewässern - Mengenanalyse und Risikobewertung. Berlin: Büro für Technikfolgen-Abschätzung beim Deutschen Bundestag (TAB), Nr. 183. (Im Erscheinen).

Koch, Klaus; Sawicki, Peter (2009): Entscheidungsunterstützung durch wissenschaftliche Evidenz. In: Public Health Forum 17 (3), S. 12.e1-12.e3.

Kümmerer, Klaus; Clark, James (2016): Green and sustainable chemistry. In: Harald Heinrichs, Pim Martens, Gerd Michelsen und Arnim Wiek (Hg.): Sustainability science. An introduction. Dordrecht: Springer, S. 43-59.

Kümmerer, Klaus; Dionysiou, Dionysios; Fatta-Kassinos, Despo (2015): Long-term strategies for tackling micropollutants. In: Dies. (Hg.): Advanced treatment technologies for urban wastewater reuse. Heidelberg: Springer, S. 291-299.

Leanza, Mathias (2010): Die Gegenwart zukünftiger Erkrankungen. Prävention und die Person. In: Bettina Paul und Henning Schmidt-Semisch (Hg.): Risiko Gesundheit. Über Risiken und Nebenwirkungen der Gesundheitsgesellschaft. Wiesbaden: VS-Verlag, S. 241-262.

Luhmann, Niklas (1988): Familiarity, confidence, trust. Problems and alternatives. In: Diego Gambetta (Hg.): Trust. Making and breaking cooperative relations. Oxford: Basil Blackwell, S. 94-107.

Offermanns, Guido (2009): Wettbewerb und Markt im Gesundheitswesen. Gegen oder für Patienten? In: Public Health Forum 17 (3), S. 7-9.

O'Grady, Laura; Witteman, Holly; Wathen, Nadine (2008): The experiential health information processing model. Supporting collaborative web-based patient education. In: BMC medical informatics and decision making 8 (1), S. 1-8. DOI: 10.1186/1472-6947-8-58.

Pahl, Ole (2015): noPILLS Project. Final Report. Interreg North-West Europe. Online verfügbar unter http://www.no-pills.eu/conference/BS_NoPills_ Final\%20Report_long_EN.pdf, zuletzt geprüft am 18.09.2018.

Paul, Bettina; Schmidt-Semisch, Henning (2010): Risiko Gesundheit. Über Risiken und Nebenwirkungen der Gesundheitsgesellschaft. Wiesbaden: VS-Verlag.

Pelikan, Jürgen (2009): Ausdifferenzierung von spezifischen Funktionssystemen für Krankenbehandlung und Gesundheitsförderung oder: Leben wir in der "Gesundheitsgesellschaft"? In: Österreichische Zeitschrift für Soziologie 34 (2), S. 28-47.

Rodrig, Svenja; Wiesemann, Hans-Olaf (2004): Der Einfluss des demographischen Wandels auf die Ausgaben der Krankenversicherung. In: Zeitschrift für die gesamte Versicherungswissenschaft 93 (1), S. 17-46.

Schlimpert, Veronika (2018): Neue US-Definition der Hypertonie in der Kritik. In: CardioVasc 18 (2), S. 16-17.
Schulte-Oehlmann, Ulrike; Oehlmann, Jörg; Püttmann, Wilhelm (2007): Humanpharmakastoffe in der Umwelt. Einträge, Vorkommen und der Versuch einer Bestandsaufnahme. In: Umweltwissenschaften und Schadstoff-Forschung Zeitschrift für Umweltchemie und Ökotoxikologie 19 (3), S. 168-179.

Shapiro, Susan (1987): The social control of impersonal trust. In: American Journal of Sociology 93 (3), S. 623-658.

Simon, Michael (2005): Das Gesundheitssystem in Deutschland. Eine Einführung in Struktur und Funktionsweise. Bern: Huber.

Skinner, Denise; Dietz, Graham; Weibel, Antoinette (2013): The dark side of trust. When Trust becomes a „poisoned chalice“. In: Organization 21 (2), S. 206-224.

Stevenson, Fiona A. (2006): The Doctor-Patient relationship. Interconnections between global processes and interaction. In: Claus Wendt und Christof Wolf (Hg.): Soziologie der Gesundheit (46. Sonderheft: Kölner Zeitschrift für Soziologie und Sozialpsychologie). Wiesbaden: VS-Verlag, S. 225-242.

Stollberg, Gunnar (2007): Kunden der Medizin? Der Mythos vom mündigen Patienten. In: Irmhild Saake und Werner Vogd (Hg.): Moderne Mythen der Medizin. Studien zur organisierten Krankenbehandlung. Berlin: Springer, S. 345-362.

Stollberg, Gunnar (2009): Das medizinische System. Überlegungen zu einem von der Soziologie vernachlässigten Funktionssystem. In: Soziale Systeme 15 (1), S. 189-217.

Toombs, Kay (1992): The meaning of illness. A phenomenological account of physician and patient. Dordrecht: Kluwer.

Touraud, Evelyne; Roig, Benoit; Sumpter, John; Coetsier, Clémence (2011): Drug residues and endocrine disruptors in drinking water: Risk for humans? In: International Journal of Hygiene and Environmental Health 214 (6), S. 437-441.

Vogd, Werner (2005): Medizinsystem und Gesundheitswissenschaften. Rekonstruktion einer schwierigen Beziehung. In: Soziale Systeme 11 (2), S. 236-270.

Webb, Simon; Ternes, Thomas; Gibert, Michel; Olejniczak, Klaus (2003): Indirect human exposure to pharmaceuticals via drinking water. In: Toxicology Letters 142 (3), S. 157-167.

Wendt, Claus (2003): Vertrauen in Gesundheitssysteme. In: Berliner Journal für Soziologie 13 (3), S. 371-393.

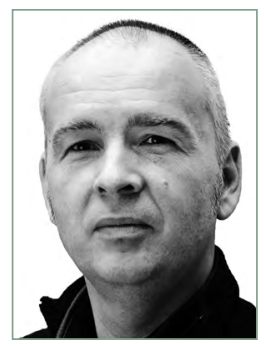

\section{DR. CHRISTIAN BÜSCHER}

ist Senior Researcher am Institut für Technikfolgenabschätzung und Systemanalyse (ITAS) am Karlsruher Institut für Technologie (KIT). 